\title{
Flavonoids in Different Parts of Lysimachia clethroides Duby Extracted by Ionic Liquid: Analysis by HPLC and Antioxidant Activity Assay
}

\author{
Jin-feng Wei, ${ }^{1,2}$ Zhi-juan Zhang, ${ }^{1}$ Li-li Cui, ${ }^{1}$ and Wen-yi Kang ${ }^{1,2}$ \\ ${ }^{1}$ Institute of Chinese Materia Medica, Henan University, Kaifeng 475004, China \\ ${ }^{2}$ Kaifeng Key Laboratory of Functional Components in Health Food, Kaifeng 475004, China \\ Correspondence should be addressed to Wen-yi Kang; kangweny@hotmail.com
}

Received 14 January 2017; Accepted 4 May 2017; Published 13 June 2017

Academic Editor: Yoshihiro Kudo

Copyright ( 92017 Jin-feng Wei et al. This is an open access article distributed under the Creative Commons Attribution License, which permits unrestricted use, distribution, and reproduction in any medium, provided the original work is properly cited.

To establish methods for simultaneous determination of isoquercitrin, astragalin in leaves, quercetin, and kaempferol in flowers of Lysimachia clethroides Duby, respectively, the methods were ultrasound-assisted extraction combined with RP-HPLC, and ionic liquid was used as the extraction solvent. Meanwhile, the antioxidant activity of the different extracts of L. clethroides was evaluated. Purospher STAR RP-C ${ }_{18}$ column $(4.6 \mathrm{~mm} \times 250 \mathrm{~mm}, 5 \mu \mathrm{m})$ was used for analysis. The flow rate was $0.6 \mathrm{~mL} \cdot \mathrm{min}^{-1}$, and the column temperature was $25^{\circ} \mathrm{C}$. The detection wavelength was $360 \mathrm{~nm}$. The mobile phases a and b consisted of acetonitrile- $0.4 \%$ phosphoric acid $(18: 82, \mathrm{v} / \mathrm{v})$, methanol (A), and 0.4\% phosphoric acid (B), respectively. Linear ranges were 0.068 1.64, 0.060 1.44, 0.0080 0.19 , and $0.0077 \sim 0.18 \mu \mathrm{g}$ for isoquercitrin, astragalin, quercetin, and kaempferol, respectively. The average recoveries of the four constituents were $99.17 \%, 98.39 \%, 100.68 \%$, and $98.81 \%$, respectively. The antioxidant activity of the extracts was detected by DPPH, ABTS, and FRAP. Under the optimized conditions, all the test solutions showed a certain antioxidant activity and the ionic liquid extracts were better than that of extract of methanol. Ionic liquid used as the extraction solvent had the potential to extract active ingredients efficiently from $L$. clethroides, and this method improved the antioxidant activity with accurate and reliable results.

\section{Introduction}

Lysimachia clethroides Duby belongs to the Primulaceae family and is abundant and widely distributed in China. The root or the whole plant of L. clethroides was used as traditional Chinese herbal medicine with many health benefits in the treatment of edema, jaundice, dysentery, rheumatic fever, amenorrhea, bruises, fractures, traumatic bleeding, mastitis, boils, and snakebite [1]. Furthermore, its tender stems and leaves serve as popular vegetable in some areas [2]. Pharmacological research showed that $L$. clethroides had many pharmacological activities such as antitumor [3, 4], antioxidant [5], hypoglycemic [6], and hepatoprotective effect [7]. The main chemical components of L. clethroides were flavonoids, triterpenes, and organic acids [8-11]. The flavonoids are responsible for the bioactivity of $L$. clethroides $[12,13]$.
Ionic liquids (ILs) are solely composed of cations and anions as liquid near room temperature [14]. ILs own a large amount of excellent properties, including negligible vapour pressure, good thermal stability, wide liquid range, good dissolving, and high electric conductivity [15]. ILs are expected to be greener alternatives to traditional volatile organic solvents with the characteristic of being environment-friendly. ILs are promising solvents for extracting the active ingredients from traditional Chinese medicine, which are not only environment-friendly but also highly efficient $[16,17]$. However, it unavoidably involved some organic solvents during the extraction. ILs in traditional Chinese medicine extraction are applied in the initial stage; it is necessary to conduct in-depth research.

Our group had reported the content of four flavonoids in L. clethroides [18], and on the basis of other studies [19-21], 
the contents of isoquercitrin, astragalin, quercetin, and kaempferol in different parts of $L$. clethroides using highperformance liquid chromatography (HPLC) were compared and a rapid, effective, and environment-friendly ionic liquid-based ultrasonic-assisted approach was established to improve the extraction yield. In addition, the antioxidant activities of the different extracts of $L$. clethroides were measured in order to prove the efficient advantage of ionic liquid in vitro.

\section{Materials and Methods}

2.1. Reagents and Materials. Isoquercitrin, astragalin, quercetin, and kaempferol of purity greater than $98 \%$ were purchased from Chengdu Pufei De Biotech Co., Ltd. Acetonitrile used for HPLC was purchased from Avantor Performance Materials, Inc. (USA). Methanol used for HPLC was purchased from Tianjin Shield Fine Chemicals Co., Ltd. The water was Wahaha pure water.

1-Butyl-3-methylimidazolium tetrafluoroborate ([BMIM]BF $\left.{ }_{4}\right)$, 1-butyl-3-methylimidazolium hexafluorophosphate ([BMIM] $\left.\mathrm{PF}_{6}\right), 1$-hexyl-3-methylimidazolium hexafluorophosphate ([HMIM] $\mathrm{PF}_{6}$ ), and 1-butyl-3-methylimidazolium bromide ([BMIM]Br) were obtained from Merck $\mathrm{KGaA}$

1,1-Diphenyl-2-picrylhydrazyl (DPPH) was obtained from Tokyo Chemical Industry Co., Ltd. 2,2'-Azino-bis(3ethylbenzothiazoline-6-sulfonic acid) (ABTS) was obtained from Fluka. 2,4,6-Tri(2-pyridinyl)-1,3,5-triazine (TPTZ), butylated hydroxytoluene (BHT), butyl-p-hydroxyanisole (BHA), and gallic acid propyl (PG) were obtained from Sigma. 6-Hydroxy-2,5,7,8-tetramethylchroman-2-carboxylic acid (Trolox) was obtained from Aldrich Chemical Co., Ltd.

Air-dried plant was collected from Huaxi District, Guizhou Province of China, in June 2014. The plant was identified as $L$. clethroides of Primulaceae family by Professor Changqin Li (Henan University, Kaifeng, China). The voucher specimens were deposited in the Institute of Natural Products, Henan University (number 20140612).

2.2. Preparation of Standard Solution. The four standard solutions of isoquercitrin, astragalin, quercetin, and kaempferol were prepared in methanol at a concentration of 1365, 1200, 160 , and $154 \mu \mathrm{g} \cdot \mathrm{mL}^{-1}$ and stored at $4^{\circ} \mathrm{C}$, respectively. The standard working solutions were prepared by appropriate dilution of the four stock standard solutions with methanol to the required concentrations. Standard working solutions (a and b) were prepared by diluting isoquercitrin and astragalin and quercetin and kaempferol stock solutions at proper concentrations, respectively.

2.3. Preparation of Test Sample Solution. Leaves $(0.50 \mathrm{~g})$ and flowers $(0.25 \mathrm{~g})$ of $L$. clethroides were mixed with $5 \mathrm{~mL}$ of [BMIM] $\mathrm{BF}_{4}$ methanol solution $\left(0.5 \mathrm{~mol} \cdot \mathrm{L}^{-1}\right)$ in glass vessel, respectively. The samples of leaves and flowers were extracted by ultrasonic-assisted extraction (20 and $45 \mathrm{~min}, 500 \mathrm{~W}$ ) and then centrifuged (4000 and $5000 \mathrm{r} \cdot \mathrm{min}^{-1}$, resp.). Then, the extract was filtrated through a $0.22 \mu \mathrm{m}$ microporous membrane. The subsequent filtrate was the test sample solutions $\mathrm{a}$ and $\mathrm{b}$ (the optimum extraction solvent, concentration of selected extraction solvent, mesh, solid-liquid ratio, ultrasonic time and power, and centrifugation speed were systematically investigated in the study).

2.4. HPLC Analysis. LC-20AT high-performance liquid chromatography (Shimadzu) was equipped with a degasser, a quaternary gradient low pressure pump, the CTO-20A column oven, a SPD-M20A UV-detector, and a SIL-20A autosampler. The data were acquired and processed using LCSolution chromatography data processing system.

The two chromatographic separations were all performed on a Purospher STAR RP-C18 column $(4.6 \mathrm{~mm} \times 250 \mathrm{~mm}$, $5 \mu \mathrm{m})$ at a column temperature of $25^{\circ} \mathrm{C}$. The flow rate of mobile phase was $0.6 \mathrm{~mL} \cdot \mathrm{min}^{-1}$. The UV detection wavelength was set at $360 \mathrm{~nm}$. All injection volumes were $10 \mu \mathrm{L}$. Mobile phase a consists of acetonitrile- $0.4 \%$ phosphoric acid $(18: 82, \mathrm{v} / \mathrm{v})$. Mobile phase $\mathrm{b}$ consists of methanol (A) and $0.4 \%$ phosphoric acid (B), and the gradient program was as follows: $0 \sim 25 \mathrm{~min}, 52 \% \mathrm{~A} ; 25 \sim 30 \mathrm{~min}, 52 \% \sim 65 \% \mathrm{~A} ; 30 \sim$ $46 \mathrm{~min}, 65 \% \mathrm{~A}$. The HPLC chromatograms of the standard solution and the extract of sample were shown in Figure 1.

2.5. Experimental Procedure. The schematic plot of the experimental procedure was shown in Figure 2.

\subsection{Antioxidant Activity In Vitro}

2.6.1. Preparation of the Sample Solution. According to the optimized conditions, methanol and ionic liquid sample solutions were prepared and concentrated to $1 \mathrm{~mL}$, respectively.

2.6.2. DPPH Assay. The sample was diluted into a range of concentrations with methanol. $10 \mu \mathrm{L}$ of sample solution (or methanol) and $175 \mu \mathrm{L}$ of DPPH methanol solution $(200 \mu \mathrm{M})$ were added to 96-well microplate and mixed. The solution was measured at $515 \mathrm{~nm}$ using a Multiskan GO microplate reader after 20 min in the dark place, with PG, BHA, and BHT as positive controls. All the experiments were performed in triplicate. The percentage of radical scavenging rate was calculated using formula (1). $\mathrm{IC}_{50}$ value was calculated from the concentration-effect linear regression curve [22].

$$
\begin{aligned}
& \text { DPPH radical scavenging rate }(\%) \\
& \quad=\left[\frac{\left(A_{0}-A_{1}\right)}{A_{0}}\right] \times 100 \%
\end{aligned}
$$

Note. $A_{0}$ was the absorbance of the DPPH itself; $A_{1}$ was the absorbance of sample and the positive control. $\mathrm{IC}_{50}$ represents the concentration of sample in $\mu \mathrm{g} \cdot \mathrm{mL}^{-1}$ which inhibits DPPH radical by $50 \%$.

2.6.3. ABTS Assay. According to literature [23], the ABTS radical working solution was prepared. Then, the sample was diluted into a range of concentrations with methanol. $10 \mu \mathrm{L}$ of sample solution (or methanol) and $200 \mu \mathrm{L}$ of ABTS solution 


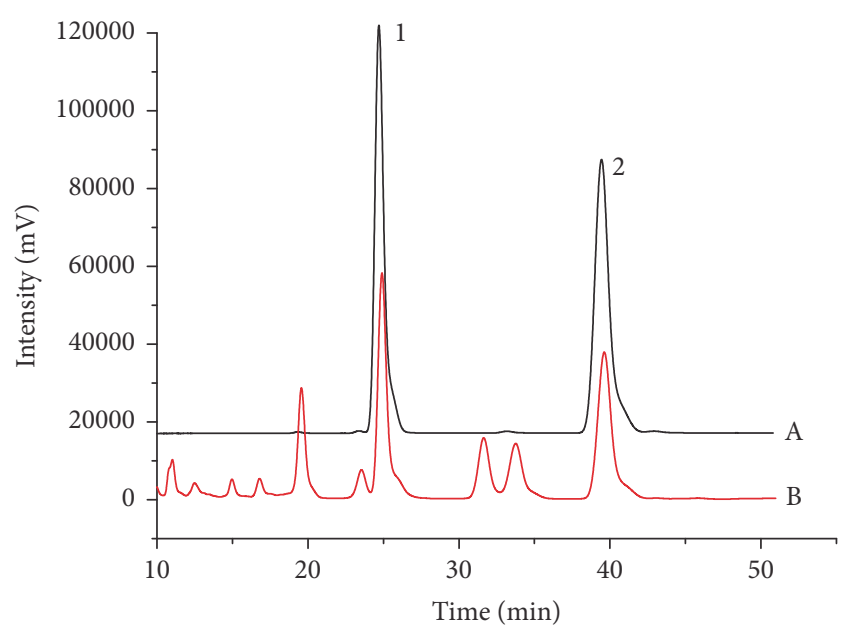

(a)

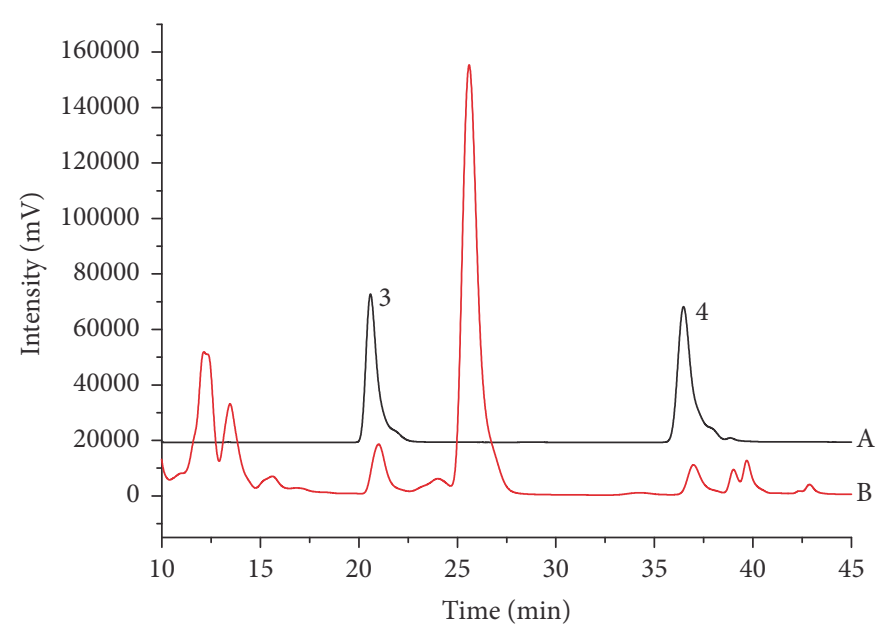

(b)

FIGURE 1: HPLC chromatograms of the standard solution (A) and the sample solution (B): (1) isoquercitrin, (2) astragalin, (3) quercetin, and (4) kaempferol.

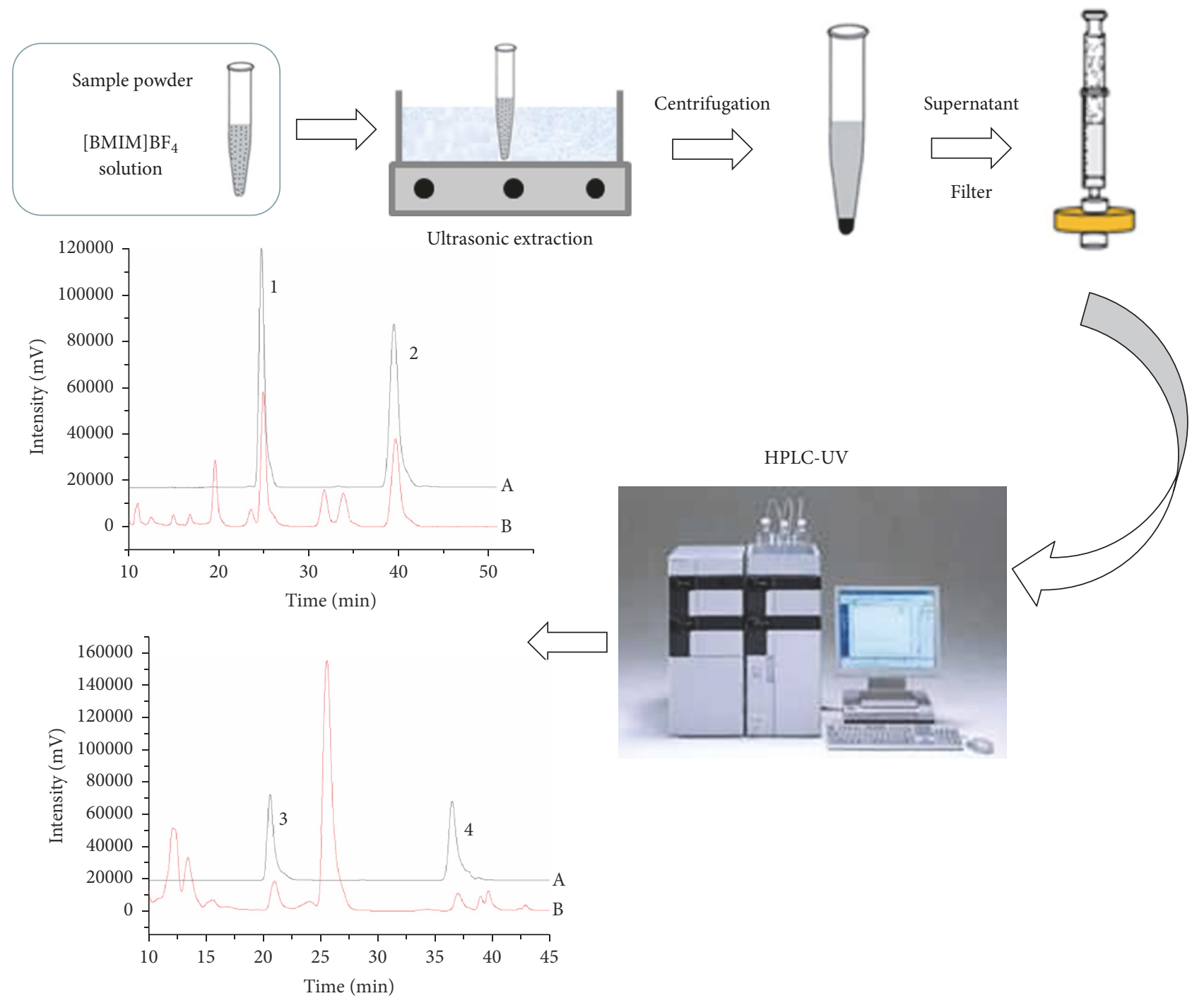

FIGURE 2: Schematic plot of the experimental procedure. 


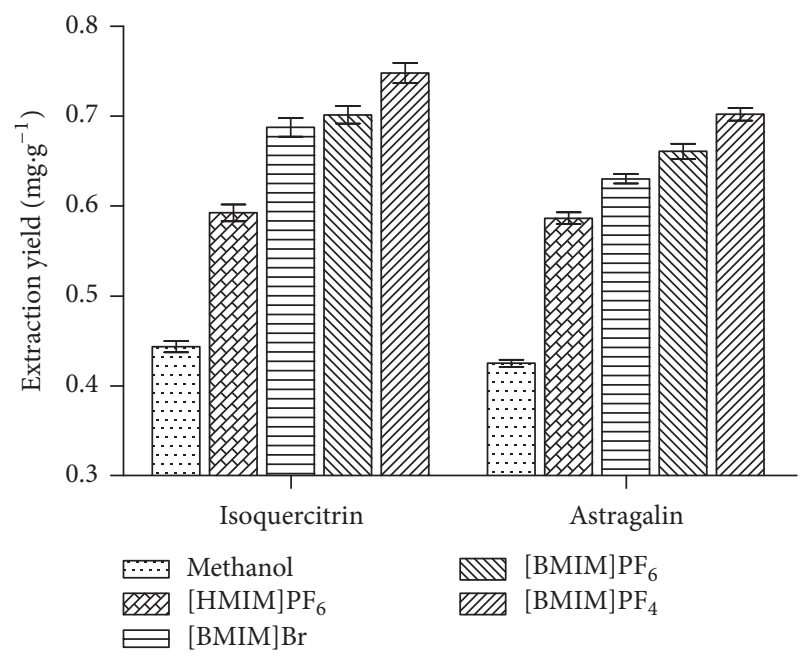

(a)

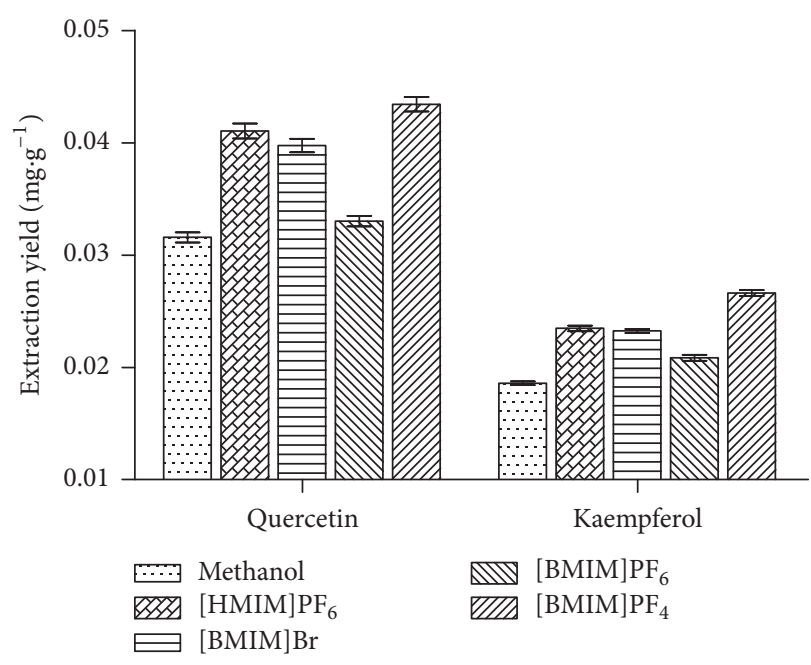

(b)

FIGURE 3: Effect of type of ILs on extraction yields. Concentration of the four ILs in methanol solution: $0.5 \mathrm{~mol} \cdot \mathrm{L}^{-1}$; the particle size: $50 \mathrm{mesh}$; solid-liquid ratio: $1: 10 \mathrm{~g} \cdot \mathrm{mL}^{-1}$; ultrasound time: $30 \mathrm{~min}$; ultrasound power: $500 \mathrm{~W}$; centrifugation speed: $5000 \mathrm{r} \cdot \mathrm{min}^{-1}$.

were added to 96-well microplate and mixed. The solution was measured at $405 \mathrm{~nm}$ after $20 \mathrm{~min}$ in the dark place. All the experiments were performed in triplicate. Calculate the percentage of radical scavenging rate and $\mathrm{IC}_{50}$ value.

2.6.4. FRAP Assay. According to literature [24], the TPTZ working solution was prepared. Then, the sample was diluted into a range of concentrations with methanol. $10 \mu \mathrm{L}$ of sample solution (or methanol) and $200 \mu \mathrm{L}$ of TPTZ solution were added to 96-well microplate and mixed. The solution was measured at $593 \mathrm{~nm}$ after $20 \mathrm{~min}$ at $37^{\circ} \mathrm{C}$. All reactions were carried out with three replications. Calculate the reducing ability of $\mathrm{Fe}^{3+}$ of the sample. The results were expressed in $\mu$ mol Trolox equivalents (TE)/g sample. The standard curve was linear when the concentration of Trolox was between 50 and $1600 \mu \mathrm{M}$. Further dilution of the sample was needed if the FRAP value measured exceeded the linear range of the standard curve.

2.7. Statistical Analysis. All the grouped data were statistically evaluated with SPSS 19.0 software. All results are expressed as mean \pm standard deviation (SD).

\section{Results and Discussion}

3.1. Optimization of Extraction Conditions. The contents of isoquercitrin, astragalin, quercetin, and kaempferol in the roots, stems, leaves, and flowers of L. clethroides were determined according to the method of literature [18]. The results showed that the contents of the four flavonoids were different, and the contents of isoquercitrin, astragalin in leaves, quercetin, and kaempferol in flowers were the highest, respectively. Therefore, the extraction conditions of isoquercitrin, astragalin in leaves, quercetin, and kaempferol in flowers of $L$. clethroides were chosen to optimize the conditions of extraction.
3.1.1. Type of ILs. The structure of IL has a significant effect on its physicochemical properties including solubility in water, the viscosity, and extraction capacity, which might have great impact on the extraction efficiency of target compounds [25]. In our study, four kinds of ILs were used as the extraction solvents, including water-soluble and water-insoluble types. In addition, methanol was selected as extraction solvent for evaluating the extraction yield compared with the developed method. In Figure 3, the addition of ILs to methanol obviously improved the extraction efficiency of the four flavonoid compounds compared with methanol as solvent. These might be due to the stronger multi-interactions between the analytes and ILs including hydrogen bonding and van der Waals interaction energy [26]. Among the four types of ILs, the extraction efficiency of $[\mathrm{BMIM}] \mathrm{BF}_{4}$ was the highest, but others did not show a certain regularity. The reason for the above phenomenon may be the fact that other ingredients in leaves and flowers had an influence on the extraction of target analytes. Therefore, $[\mathrm{BMIM}] \mathrm{BF}_{4}$ was chosen as the component of extraction solvent in the following studies.

3.1.2. $[B M I M] B F_{4}$ Concentration. The concentration effect of $[\mathrm{BMIM}] \mathrm{BF}_{4}$ on the extraction was shown in Figure 4. The extraction yields continued to rise when the concentration increased from 0.1 to $0.5 \mathrm{~mol} \cdot \mathrm{L}^{-1}$ and then different degrees of decrease appeared with the further increase of the concentration. This was because the solubility of the ionic liquid solution increased with the increasing concentration, but the diffusion and transfer capability of extraction solvent decreased, which led to declining of the solvent's penetration power into the interior of sample matrix [27, 28].

3.1.3. Particle Size. The effect of the particle sizes of sample on extraction efficiency was investigated. In Figure 5, the highest extraction yields of isoquercitrin, astragalin in leaves, quercetin, and kaempferol in flowers were achieved at 24 and 


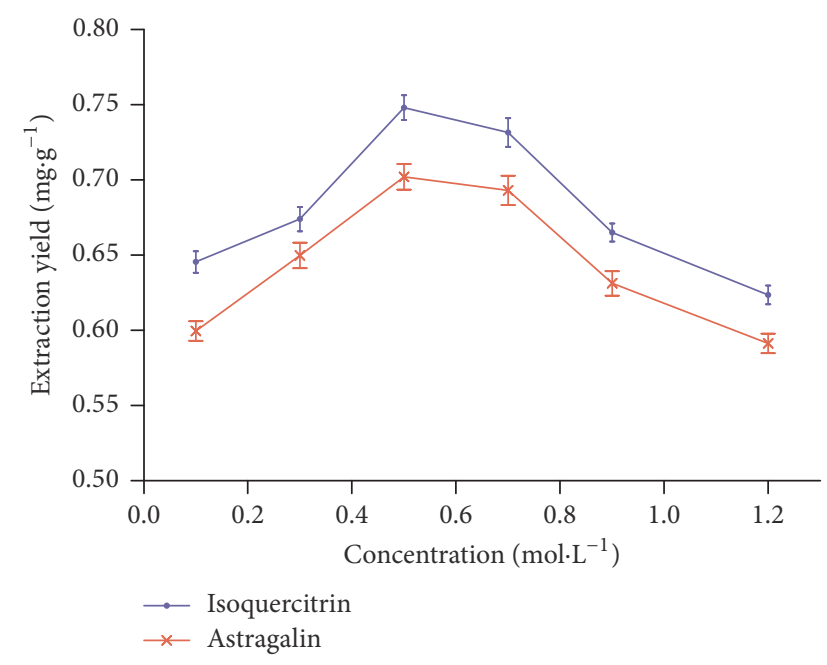

(a)

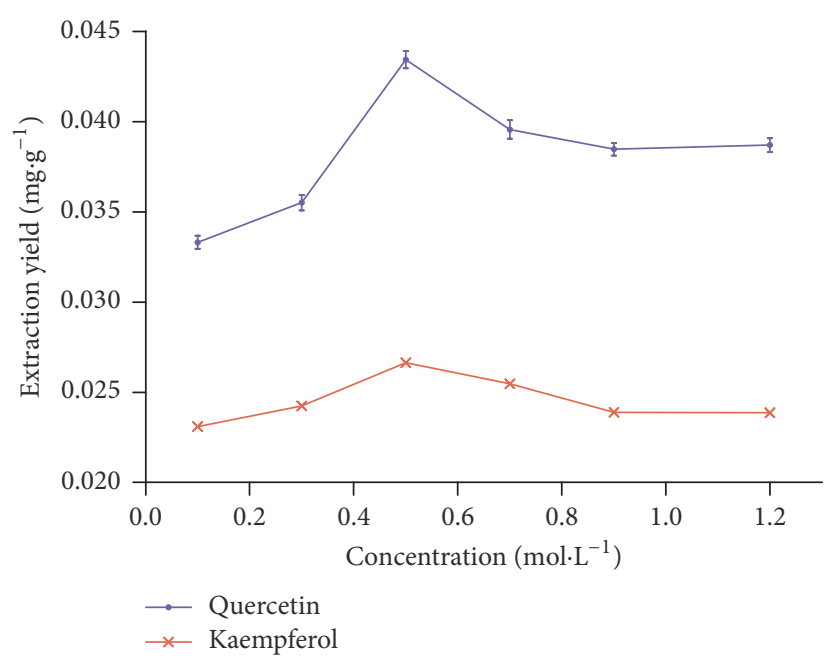

(b)

FIGURE 4: Effect of the concentration of IL on extraction yields. Type of IL: [BMIM]BF 4 ; the particle size: 50 mesh; solid-liquid ratio: $1: 10 \mathrm{~g} \cdot \mathrm{mL}^{-1}$; ultrasound time: $30 \mathrm{~min}$; ultrasound power: $500 \mathrm{~W}$; centrifugation speed: $5000 \mathrm{r} \cdot \mathrm{min}^{-1}$.

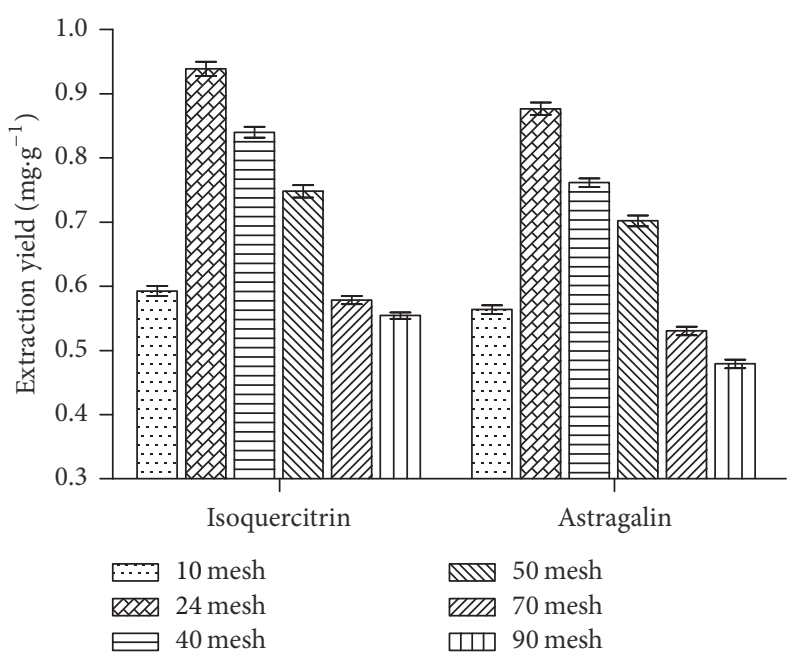

(a)

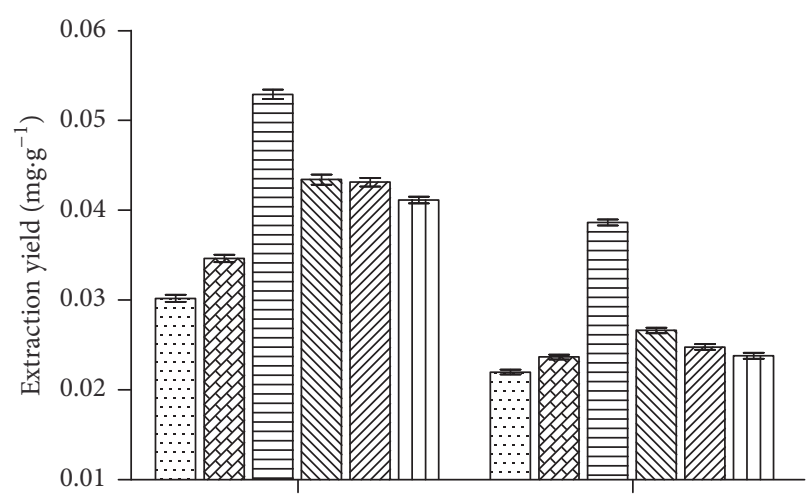

Quercetin

Kaempferol

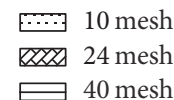

50 mesh VIn 70 mesh पाण 90 mesh

(b)

FIGURE 5: Effect of the particle size on extraction yields. Type of IL: $[\mathrm{BMIM}] \mathrm{BF}$; concentration: $0.5 \mathrm{~mol} \cdot \mathrm{L}^{-1}$; solid-liquid ratio: $1: 10 \mathrm{~g} \cdot \mathrm{mL}^{-1}$; ultrasound time: $30 \mathrm{~min}$; ultrasound power: $500 \mathrm{~W}$; centrifugation speed: $5000 \mathrm{r} \cdot \mathrm{min}^{-1}$.

40 mesh, respectively. It might be the smaller the particle size, the easier the extraction, but the samples of leaves and flowers can easily be conglomerated by ionic liquid when the particle size was too small [29]. In addition, the different optimum particle sizes of leaves and flowers might be caused by the difference of organizational structures.

3.1.4. Solid-Liquid Ratio. Six different ratios of solid-liquid $\left(1: 5,1: 10,1: 20,1: 30,1: 50\right.$, and $\left.1: 80 \mathrm{~g} \cdot \mathrm{mL}^{-1}\right)$ were chosen to evaluate the effect of solid-liquid ratio on the extraction yield. The results in Figure 6 indicated that the maximum extraction rates of isoquercitrin, astragalin in leaves, quercetin, and kaempferol in flowers were obtained at $1: 10$ and $1: 20 \mathrm{~g} \cdot \mathrm{mL}^{-1}$, respectively. Results showed that the target analytes in the sample had basically been extracted. When the proportion of extraction agent was increased, the extraction efficiencies were constant or decreased slightly; this finding might be related to the viscosity of ionic liquid.

3.1.5. Ultrasound Time. To investigate the influence of ultrasound time on the extraction efficiency, six time points ranging from 10 to $60 \mathrm{~min}$ were chosen. In Figure 7, the maximum extraction efficiencies of isoquercitrin, astragalin in leaves, quercetin, and kaempferol in flowers were obtained at 20 and $45 \mathrm{~min}$, respectively. Nevertheless, the ultrasound time was further extended; the extraction yields of target 


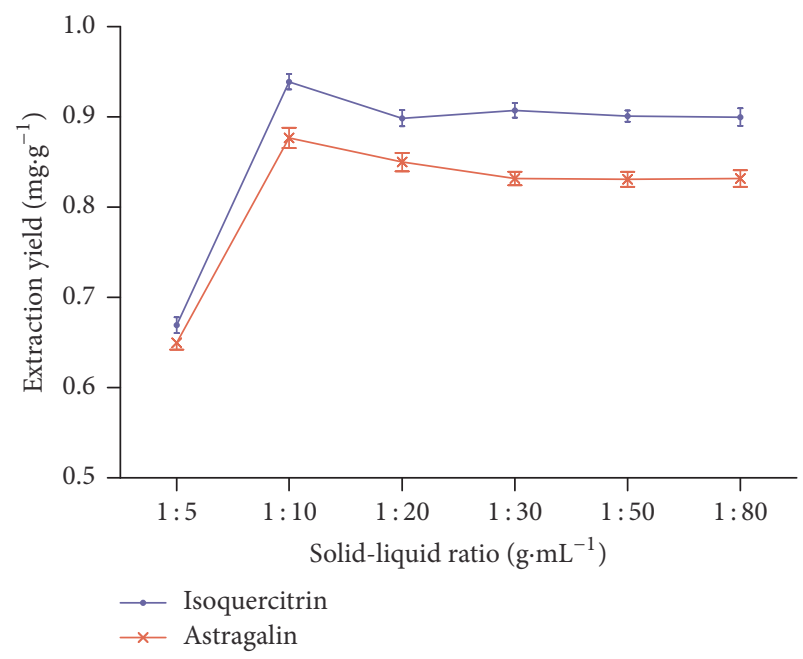

(a)

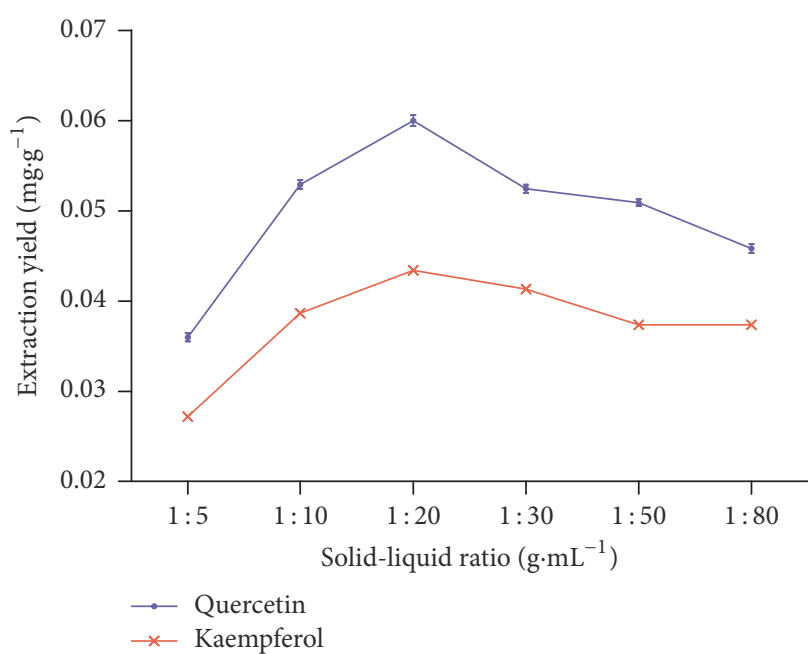

(b)

FiguRE 6: Effect of solid-liquid ratio on extraction yields. Type of IL: [BMIM]BF ; concentration: 0.5 mol. $\mathrm{L}^{-1}$; the particle size: 24 mesh (a) and 40 mesh (b); ultrasound time: $30 \mathrm{~min}$; ultrasound power: $500 \mathrm{~W}$; centrifugation speed: $5000 \mathrm{r} \cdot \mathrm{min}^{-1}$.

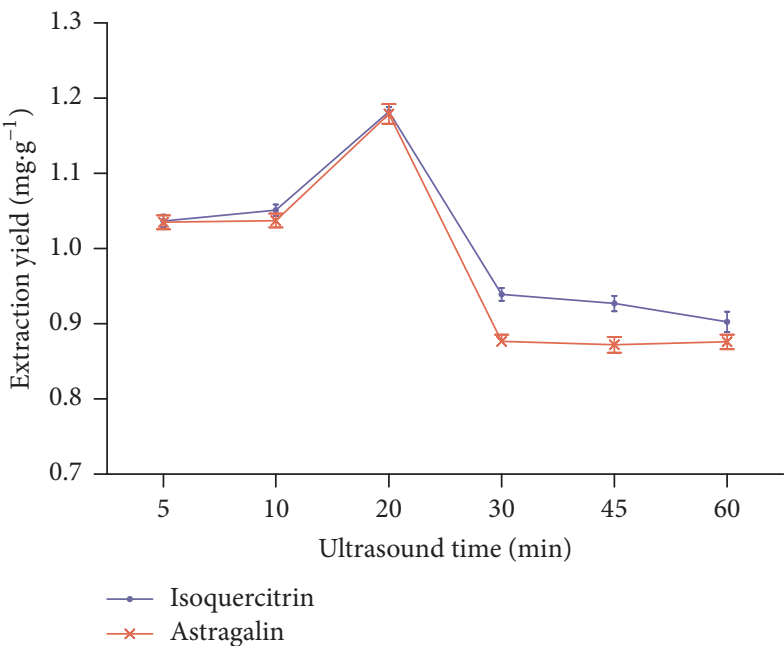

(a)

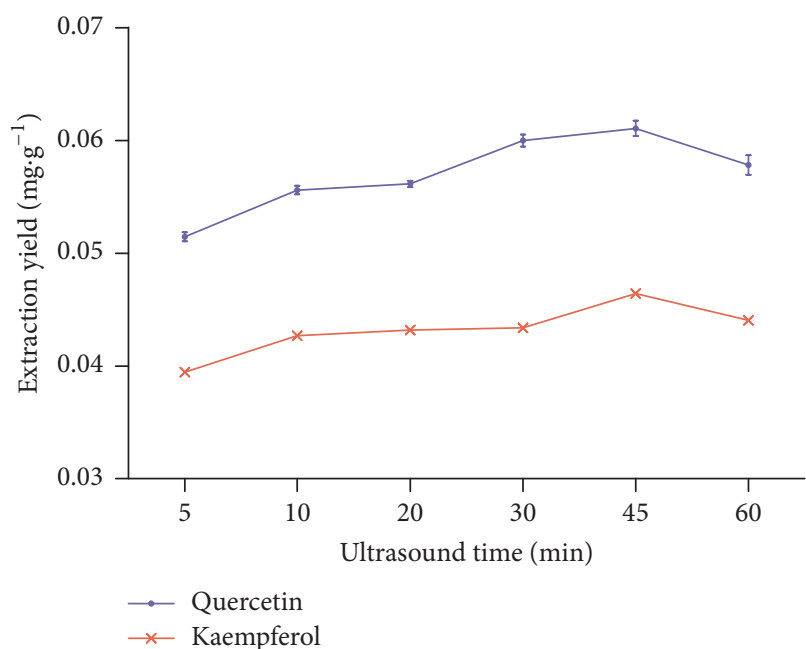

(b)

FIGURE 7: Effect of ultrasound time on extraction yields. Type of IL: [BMIM]BF ${ }_{4}$; concentration: $0.5 \mathrm{~mol} \cdot \mathrm{L}^{-1}$; the particle size: $24 \mathrm{mesh}(\mathrm{a})$ and 40 mesh (b); solid-liquid ratio: 1:10 g. $\mathrm{mL}^{-1}$ (a) and $1: 20 \mathrm{~g} \cdot \mathrm{mL}^{-1}$ (b); ultrasound power: $500 \mathrm{~W}$; centrifugation speed: $5000 \mathrm{r} \cdot \mathrm{min}^{-1}$.

analytes had a certain decrease; the reason for the above phenomenon may be the fact that abundant sonication heat destroys the structure of the analytes [30].

3.1.6. Ultrasonic Power. The ultrasonic power had a significant effect on the extraction of the analytes. In our study, the effect of different ultrasonic powers on extraction efficiency was investigated from 200 to $500 \mathrm{~W}$ (in Figure 8). Results showed that the extraction efficiencies were increased with the increase of ultrasonic power. Therefore, $500 \mathrm{~W}$ was chosen as ultrasonic power in this study.

3.1.7. Centrifugation Speed. The influence of centrifugation speed on the extraction yield of the target analyte was investigated. The centrifugation time was set at $5 \mathrm{~min}$, which was a reasonable time for productivity of the method [31]. In Figure 9, results showed that the maximum extraction yields of isoquercitrin, astragalin in leaves, quercetin, and kaempferol in flowers were obtained at 4000 and $5000 \mathrm{r} \cdot \mathrm{min}^{-1}$, respectively. The reason might be that the higher centrifugal speed affects the solubility of the analytes, so that the solubility of the analytes is reduced.

\subsection{Method Validation}

3.2.1. System Suitability Test (SST). System suitability test (SST) of HPLC includes the theoretical plate number $(n)$, the resolution (Rs), the tailing factor $(T)$, and the repeatability. 


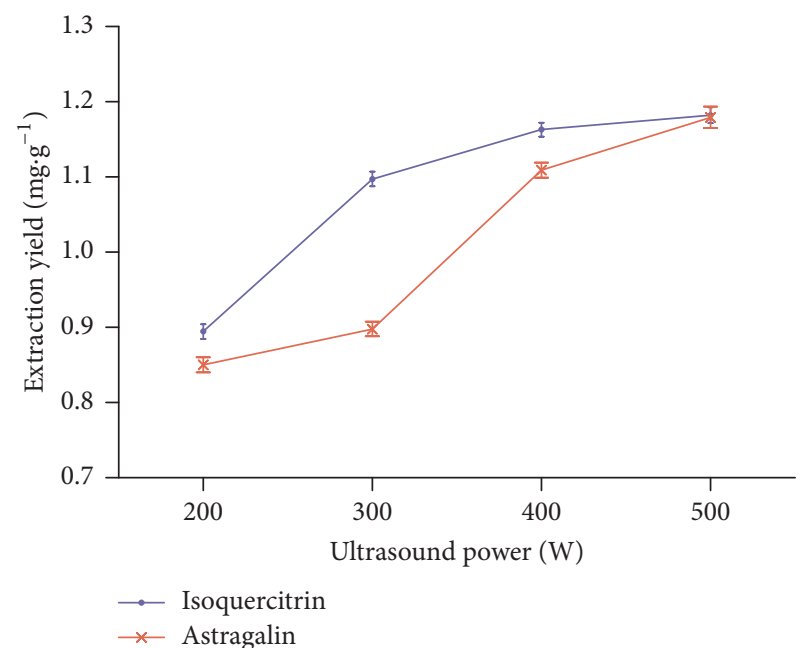

(a)

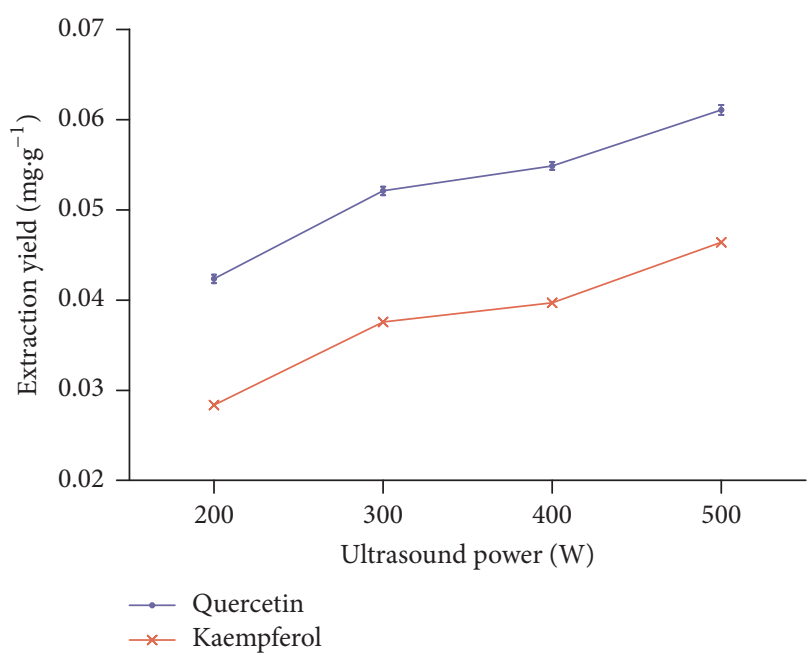

(b)

FIGURE 8: Effect of ultrasound power on extraction yields. Type of IL: $[\mathrm{BMIM}] \mathrm{BF}_{4}$; concentration: $0.5 \mathrm{~mol} \cdot \mathrm{L}^{-1}$; the particle size: 24 mesh (a) and 40 mesh (b); solid-liquid ratio: $1: 10 \mathrm{~g} \cdot \mathrm{mL}^{-1}$ (a) and 1:20 g. $\mathrm{mL}^{-1}$ (b); ultrasound time: $20 \mathrm{~min}$ (a) and 45 min (b); centrifugation speed: $5000 \mathrm{r} \cdot \mathrm{min}^{-1}$.

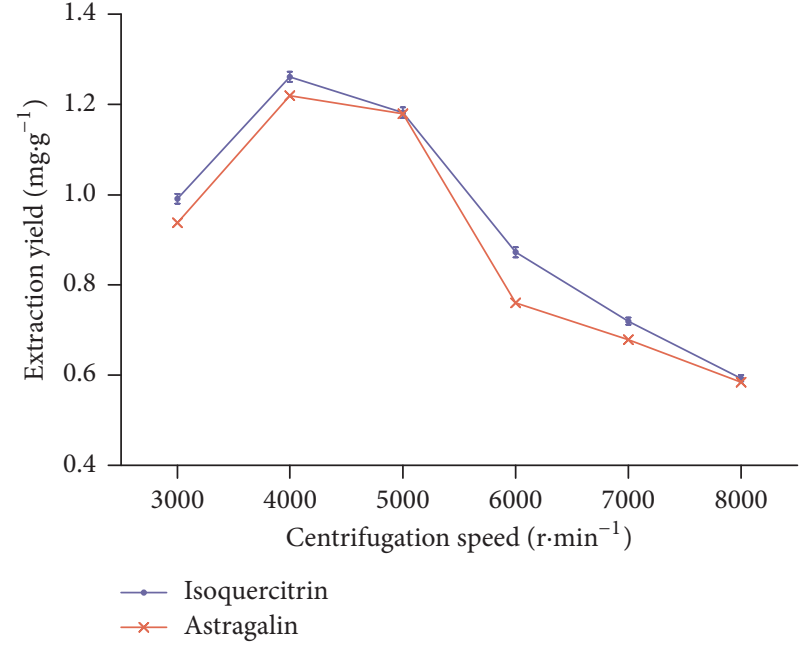

(a)

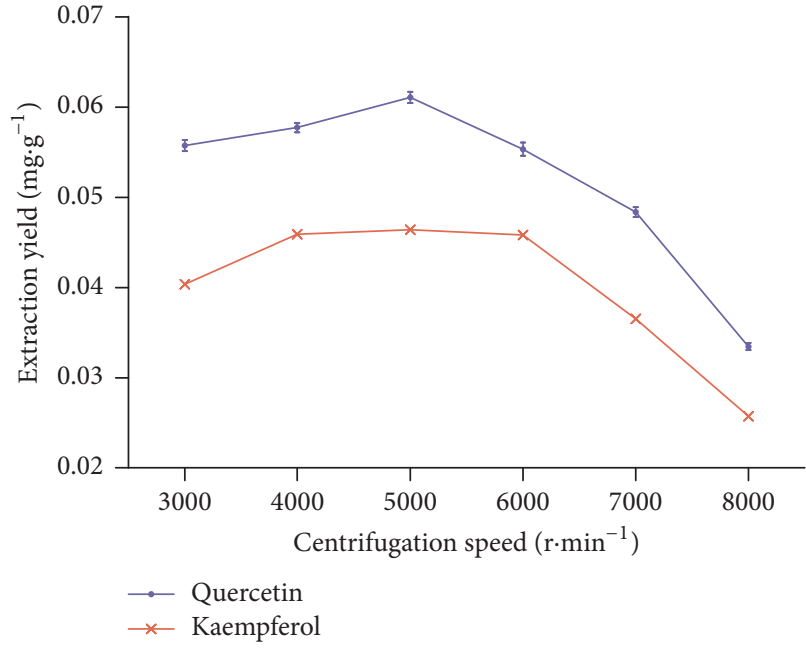

(b)

FIGURE 9: Effect of centrifugation speed on extraction yields. Type of IL: [BMIM]BF ; concentration: $0.5 \mathrm{~mol} \cdot \mathrm{L}^{-1}$; the particle size: $24 \mathrm{mesh}$ (a) and 40 mesh (b); solid-liquid ratio: $1: 10 \mathrm{~g} \cdot \mathrm{mL}^{-1}$ (a) and $1: 20 \mathrm{~g} \cdot \mathrm{mL}^{-1}$ (b); ultrasound time: $20 \mathrm{~min}$ (a) and 45 min (b); ultrasound power: $500 \mathrm{~W}$.

The repeatability was represented by the relative standard deviation of the peak area (RSD\%). The results of SST were shown in Table 1, which shows that the chromatographic systems had good suitability.

3.2.2. Linearity. The stock standard solutions $\mathrm{a}$ and $\mathrm{b}$ were accurately injected $0.5,1,2,4,6,8,10$, and $12 \mu \mathrm{L}$ to chromatographic instrument for the construction of calibration curves, respectively. The calibration curves were constructed by plotting the peak areas versus the injection volume of each compound. The results were demonstrated in Table 2. $r$ values were in the range from 0.9996 to 1.0000 , which indicated that the methods had good linearity.
3.2.3. Precision. The stock standard solutions $\mathrm{a}$ and $\mathrm{b}$ were accurately injected $10 \mu \mathrm{L}$ to chromatographic instrument, respectively. And they were analyzed in six replicates within one day for determining the precision of the developed assay. The RSDs of peak areas for isoquercitrin, astragalin, quercetin, and kaempferol were $0.46 \%, 0.83 \%, 0.23 \%$, and $0.24 \%$, respectively. The results indicated that the methods were precise for quantitative analysis of isoquercitrin, astragalin, quercetin, and kaempferol.

3.2.4. Stability. The sample solutions were prepared under the optimum extraction conditions and placed at room temperature and then were injected $10 \mu \mathrm{L}$ to chromatographic 
TABLE 1: System suitability test (SST) of HPLC.

\begin{tabular}{lcccc}
\hline Compound & Theoretical plate number & Resolution & Tailing factor & Repeatability \\
\hline Isoquercitrin & 8392 & 1.52 & 1.31 & 1.18 \\
Astragalin & 8834 & 10.95 & 1.25 & 0.87 \\
Quercetin & 7071 & 5.50 & 1.35 & 1.13 \\
Kaempferol & 13956 & 8.54 & 1.35 & 1.02 \\
\hline
\end{tabular}

TABLE 2: Regression equations and linear ranges for four compounds.

\begin{tabular}{lccc}
\hline Compound & Regressive equation & $r$ & Linear range/ $\mu \mathrm{g}$ \\
\hline Isoquercitrin & $Y=3.19 \times 10^{6} X-2.92 \times 10^{4}$ & 0.9996 & $0.068 \sim 1.64$ \\
Astragalin & $Y=3.00 \times 10^{6} X-2.32 \times 10^{4}$ & 0.9997 & $0.060 \sim 1.44$ \\
Quercetin & $Y=7.42 \times 10^{6} X-9.58 \times 10^{3}$ & 1.0000 & $0.0080 \sim 0.19$ \\
Kaempferol & $Y=8.90 \times 10^{6} X-1.49 \times 10^{4}$ & 1.0000 & $0.0077 \sim 0.18$ \\
\hline
\end{tabular}

$Y$ : peak area; $X$ : injection amount $(\mu \mathrm{g})$.

TABLE 3: Antioxidant activity of the different extracts of $L$. clethroides.

\begin{tabular}{|c|c|c|c|c|c|}
\hline \multirow{2}{*}{ Sample } & \multicolumn{2}{|l|}{$\mathrm{DPPH}$} & \multicolumn{2}{|l|}{ ABTS } & \multirow{2}{*}{$\begin{array}{c}\text { FRAP } \\
\text { TEAC/ } \mu \mathrm{mol} \cdot \mathrm{g}^{-1}\end{array}$} \\
\hline & Radical scavenging rate/\% & $\mathrm{IC}_{50} / \mu \mathrm{g} \cdot \mathrm{mL}^{-1}$ & Radical scavenging rate/\% & $\mathrm{IC}_{50} / \mu \mathrm{g} \cdot \mathrm{mL}^{-1}$ & \\
\hline Leaf's methanol extract & 93.91 & $1403 \pm 80$ & 97.01 & $144 \pm 5$ & $38.58 \pm 0.72$ \\
\hline Leaf's ionic liquid extract & 88.48 & $638 \pm 60$ & 93.23 & $50 \pm 2$ & $105.79 \pm 7.02$ \\
\hline Flower's methanol extract & 93.92 & $1004 \pm 12$ & 98.04 & $151 \pm 1$ & $50.69 \pm 1.10$ \\
\hline Flower's ionic liquid extract & 89.77 & $460 \pm 36$ & 96.36 & $51 \pm 2$ & $105.83 \pm 1.25$ \\
\hline$[\mathrm{BMIM}] \mathrm{BF}_{4}$ & -4.43 & NT & 0.03 & NT & NT \\
\hline $\mathrm{BHA}$ & 76.00 & $15 \pm 1.46$ & 99.94 & $2.06 \pm 0.08$ & $5919.11 \pm 14.10$ \\
\hline BHT & 73.06 & $12 \pm 0.48$ & 99.42 & $6.56 \pm 0.10$ & $589.20 \pm 59.20$ \\
\hline PG & 95.61 & $4.01 \pm 0.08$ & 95.80 & $0.69 \pm 0.05$ & $9441.33 \pm 69.27$ \\
\hline
\end{tabular}

Note. NT means not available because of low activity; BHA, BHT, and PG are positive controls.

instrument at $0,3,6,9,12$, and $24 \mathrm{~h}$, respectively. The RSDs of peak areas for isoquercitrin, astragalin, quercetin, and kaempferol were $0.67 \%, 0.83 \%, 1.65 \%$, and $1.74 \%$. The results indicated that the sample solution was basically stable at room temperature within $24 \mathrm{~h}$.

3.2.5. Repeatability. Six test sample solutions a and b were processed under the optimum extraction conditions and then injected $10 \mu \mathrm{L}$ to chromatographic instrument for analysis. The RSDs of peak areas for the four compounds were $2.03 \%, 1.84 \%, 1.15 \%$, and $1.40 \%$, indicating that the analytical methods had an acceptable level of repeatability.

3.2.6. Recovery. Nine batches of leaves and flowers samples of $L$. clethroides were prepared and divided into three groups, respectively. Then the four standard substances at three different amounts were added to the leaves and flowers samples. The spiked samples were prepared according to the optimum extraction conditions. All the calculated recovery values of the analytes ranged from 98.39 to $100.68 \%$ and the RSDs were less than $2.20 \%$. The results demonstrated that the methods were reasonable and feasible.

3.3. Antioxidant Activity of the Different Extracts of $L$. clethroides In Vitro. The antioxidant activities of methanol and ionic liquid sample solutions were evaluated by the DPPH, ABTS, and FRAP assay comprehensively, and the results were shown in Table 3. Results showed that the ionic liquid extract had stronger antioxidant activity than that of the methanol extract, and the ionic liquid itself had no antioxidant activity. Results indicated that the ionic liquid used as extraction solvent had more advantages for extracting ingredients.

3.4. Sample Analysis. Ionic liquid was successfully applied for the determination of isoquercitrin, astragalin in leaves, quercetin, and kaempferol in flowers of L. clethroides by the optimized conditions, respectively. The mass fractions of isoquercitrin, astragalin in leaves, quercetin, and kaempferol in flowers were $1.262,1.219,0.061$, and $0.046 \mathrm{mg} \cdot \mathrm{g}^{-1}(n=3)$, respectively.

\section{Conclusion}

The method ionic liquid-based ultrasonic-assisted extraction had been developed for the extraction of isoquercitrin, astragalin in leaves, quercetin, and kaempferol in flowers of $L$. clethroides. Compared with the conventional extraction with methanol, the proposed approaches provided higher extraction efficiencies. Results demonstrated that the methods 
were simple, rapid, green, and effective. ILs can be recycled by some methods, such as vacuum distillation, membrane filtration, salting out, and liquid-liquid extraction [32]. Considering the unique properties of ILs, the methods have a great promising prospect in sample preparation of Chinese herbal medicine.

\section{Conflicts of Interest}

The authors declare that there are no conflicts of interest.

\section{Authors' Contributions}

Wen-yi Kang and Jin-feng Wei conceived the research idea. Zhi-juan Zhang and Li-li Cui conducted the experiments, collected the plant specimens, analyzed and interpreted the data, and prepared the first draft. Wen-yi Kang, Zhi-juan Zhang, and Jin-feng Wei critically read and revised the paper. All the authors read and approved the paper before its final submission.

\section{Acknowledgments}

This work was supported by Henan Province University Science and Technology Innovation Team (16IRTSTHN019), Science and Technology Innovation Team of Kaifeng City (2016-124), Kaifeng City Science and Technology Innovation Talent (1509010), and Key project in Science and Technology Agency of Kaifeng (1603111).

\section{References}

[1] X. M. Hu, W. K. Zhang, and Q. S. Zhu, The Chinese Materia Medica. Shanghai: Shanghai Science and Technology Press, 1999.

[2] Z.-J. Zhang, Z.-Y. Xia, J.-M. Wang, X.-T. Song, J.-F. Wei, and W.Y. Kang, "Effects of flavonoids in Lysimachia clethroides duby on the activities of cytochrome P450 CYP2E1 and CYP3A4 in rat liver microsomes," Molecules, vol. 21, no. 6, article no. 738, 2016.

[3] L. H. Tang, X. Y. Xu, B. G. You, W. Zhang, and Y. Q. Wang, "Anti-tumor effect and its mechanism of total flavones of Lysimachia clethroides Duby," Shanghai Journal of Traditional Chinese Medicine, vol. 41, no. 5, pp. 74-76, 2007.

[4] Q.-M. Xu, Y.-L. Liu, Y.-L. Feng, L.-H. Tang, and S.-L. Yang, "A new e-ring $\gamma$-lactone pentacyclic triterpene from lysimachia clethroides and its cytotoxic activities," Chemistry of Natural Compounds, vol. 48, no. 4, pp. 597-600, 2012.

[5] C. F. Li, Y. L. Song, Y. X. Liu, and W. Y. Kang, "Antioxidant activity of extracts from Lysimachia clethroides," Fine Chemicals, vol. 25, pp. 1191-1193, 2008.

[6] J. F. Wei, "Study on Chemical Constitutens, Hepatoprotective and Antihyperglycemic Effect of Lysimachia paridiformis var. stenophylla and Lysimachia clethroides," MSD thesis, 2012, Henan University, Kaifeng.

[7] D. Liang, "Study on the chemical composition and biological activity of Lysimachia clethroides and chemical conversion of synthetic," MSD thesis, 2012, Peking Union Medical College, Beijing.
[8] S. M. Yue, B. Q. Chen, P. F. Yuan, W. H. Cui, and W. Y. Kang, "Chemical Constituents of Lysimachia clethroides Duby," Chinese Phramaceutical Journal, vol. 46, pp. 341-343, 2011.

[9] L. F. Ding, Y. D. Guo, X. D. Wu, and Y. H. Ma, "Chemical constituents, of flavonoids in Lysimachia clethroides," Chinese Traditional Patent Medicine, vol. 32, no. 05, pp. 827-830, 2010.

[10] Q. M. Xu, L. H. Tang, X. Li, L. L. Hao, X. R. Li, and S. L. Yang, "Isolation and Identification of 3-O-p-Coumaroyloxyl Pentacyclic Triterpenoids from Lysimachia clethroides Duby," Chin Pharm J, vol. 45, pp. 825-828, 2010.

[11] H. Y. Zou and P. F. Tu, "Studies on the chemical constituents of Lysimachia clethroides Duby," Chin Tradit Herb Drugs, vol. 40, pp. 704-708, 2009.

[12] L. H. Tang, X. Y. Xu, B. G. You, Y. Q. Wang, and W. Zhang, "Effect of flavones from Lysimachia clethroides on gene expresion of HL-60 cell," Pharm Clin Res, vol. 15, pp. 21-24, 2007.

[13] Y. Q. Wang, L. H. Tang, Z. Q. Liang, and Z. L. Gu, "Primary studies on the anti-uterine cervix cancer effects of the extract ZE4 from Lysimachia clethroides Duby," Chin Pharmacol Bull, vol. 23, pp. 925-929, 2007.

[14] A. A. Aerov, A. R. Khokhlov, and I. I. Potemkin, "Why ionic liquids can possess extra solvent power," Journal of Physical Chemistry B, vol. 110, no. 33, pp. 16205-16207, 2006.

[15] J. F. Wei, P. R. Cao, J. M. Wang, and W. Y. Kang, "Analysis of tilianin and acacetin in Agastache rugosa by high-performance liquid chromatography with ionic liquids-ultrasound based extraction," Chemistry Central Journal, vol. 10, p. 76, 2016.

[16] W. Y. Ma, Y. B. Lu, R. L. Hu, J. H. Chen, Z. Z. Zhang, and Y. J. Pan, "Application of ionic liquids based microwave-assisted extraction of three alkaloids $\mathrm{N}$-nornuciferine, $\mathrm{O}$-nornuciferine, and nuciferine from lotus leaf," Talanta, vol. 80, no. 3, pp. 12921297, 2010.

[17] W. Liu, D. D. Li, H. S. Yang et al., "Determination of oleanic acid and paeoniflorin in Paeonia lactiflora by ultrasound-assisted ionic liquid-reversed phase liquid chromatography," China J Chin Mater Med, vol. 40, pp. 443-449, 2015.

[18] J. F. Wei, Z. J. Zhang, D. D. Li, W. Liu, and W. Y. Kang, "Analysis of four flavonoids in Lysimachia clethroides using ionic liquidassisted extraction," China J Chin Mater Med, vol. 40, pp. 13051310, 2015.

[19] J. F. Wei, Z. H. Yin, and F. D. Shang, "Volatiles in the Lysimachia clethroides Duby by head space solid phase microextraction coupled with gas chromatography-mass spectrometry (HSSPME-GC-MS)," African Journal of Pharmacy and Pharmacology, vol. 6, pp. 2484-2487, 2012.

[20] J. F. Wei, Y. Y. Li, Z. H. Yin, F. Gong, and F. D. Shang, "Antioxidant activities in vitroand hepatoprotective effects of Lysimachia clethroides Duby on $\mathrm{CCl}_{4}$-induced acute liver injury in mice," Afr J Pharm Pharmacol, vol. 6, pp. 743-750, 2012.

[21] J. F. Wei, X. Chang, W. Wang, and W. Y. Kang, "Chemical constituents from Lysimachia circaeoides," Journal of Chinese Medicine Material, vol. 36, pp. 1441-1443, 2013.

[22] W.-Y. Kang, Y.-L. Song, and L. Zhang, " $\alpha$-Glucosidase inhibitory and antioxidant properties and antidiabetic activity of Hypericum ascyron L.," Medicinal Chemistry Research, vol. 20, no. 7, pp. 809-816, 2011.

[23] W.-Y. Kang, C.-F. Li, and Y.-X. Liu, "Antioxidant phenolic compounds and flavonoids of Mitragyna rotundifolia (Roxb.) Kuntze in vitro," Medicinal Chemistry Research, vol. 19, no. 9, pp. 1222-1232, 2010. 
[24] W. Kang and J. Wang, "In vitro antioxidant properties and in vivo lowering blood lipid of Forsythia suspense leaves," Medicinal Chemistry Research, vol. 19, no. 7, pp. 617-628, 2010.

[25] C. F. Poole and S. K. Poole, "Extraction of organic compounds with room temperature ionic liquids," Journal of Chromatography A, vol. 1217, no. 16, pp. 2268-2286, 2010.

[26] Z. Guo, B. Lue, K. Thomasen, A. S. Meyer, and X. Xu, "Predictions of flavonoid solubility in ionic liquids by COSMO-RS: experimental verification, structural elucidation, and solvation characterization," Green Chemistry, vol. 9, no. 12, pp. 1362-1373, 2007.

[27] S. Y. Dong, Q. Hu, J. Li, Y. L. Fu, Y. Q. Xing, and T. L. Huang, "Ionic liquid-based microwave-assisted extraction followed high-performance liquid chromatography for the simultaneous determination of five organic oxygen pesticides in soil samples," Chinese Journal of Analysis Laboratory, vol. 32, pp. 48-52, 2013.

[28] H. Wu, M. Chen, Y. Fan, F. Elsebaei, and Y. Zhu, "Determination of rutin and quercetin in Chinese herbal medicine by ionic liquid-based pressurized liquid extraction-liquid chromatography- chemiluminescence detection," Talanta, vol. 88, pp. 222-229, 2012.

[29] R. Jin, L. Fan, and X. An, "Ionic liquid-assisted extraction of paeonol from cynanchum paniculatum," Chromatographia, vol. 73, no. 7-8, pp. 787-792, 2011.

[30] P. Liang, F. Wang, and Q. Wan, "Ionic liquid-based ultrasoundassisted emulsification microextraction coupled with high performance liquid chromatography for the determination of four fungicides in environmental water samples," Talanta, vol. 105, pp. 57-62, 2013.

[31] C. K. Zacharis, C. Christophoridis, and K. Fytianos, "Vortexassisted liquid-liquid microextraction combined with gas chromatography-mass spectrometry for the determination of organophosphate pesticides in environmental water samples and wines," Journal of Separation Science, vol. 35, no. 18, pp. 2422-2429, 2012.

[32] H. Liu, X. Y. Wei, J. H. Li, T. Li, and F. Wang, "Review of ionic liquids recycling," Journal of Cellulose Science Technology, vol. 21, pp. 63-69, 2013. 

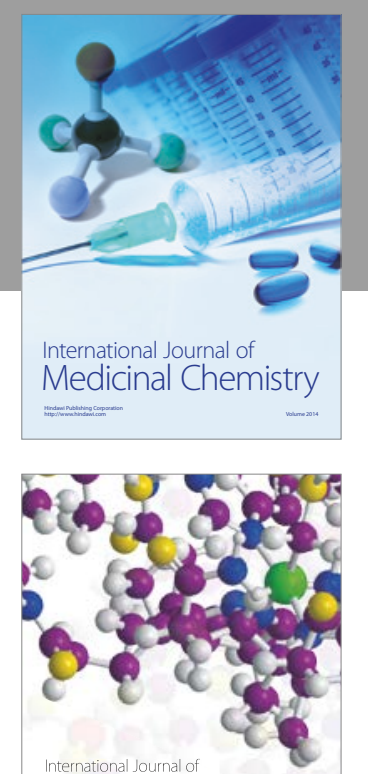

Carbohydrate Chemistry

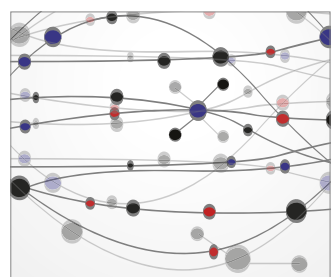

The Scientific World Journal
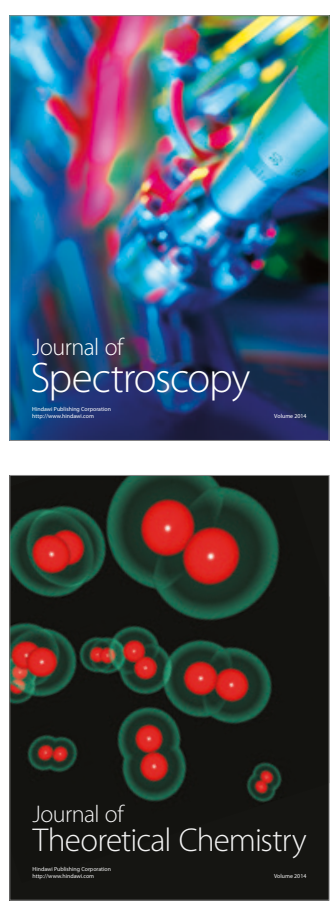
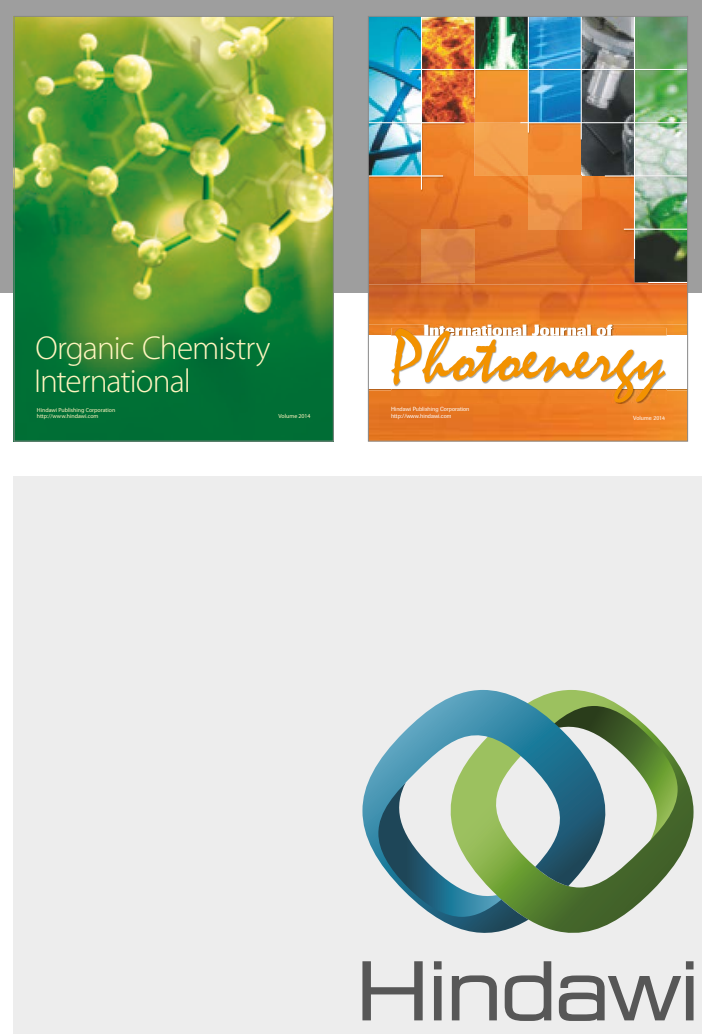

Submit your manuscripts at

https://www.hindawi.com

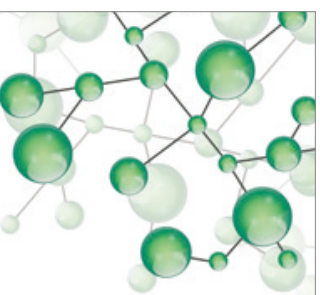

International Journal of

Inorganic Chemistry

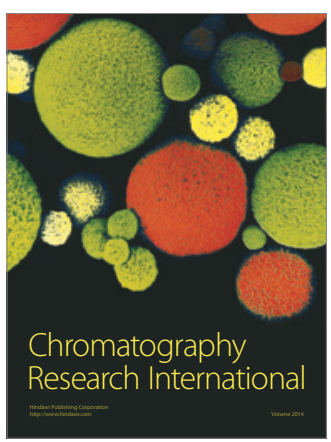

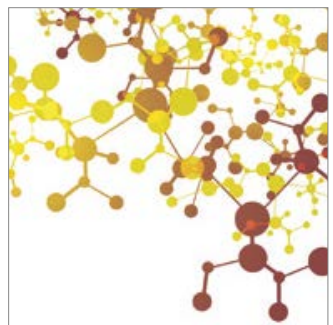

Applied Chemistry
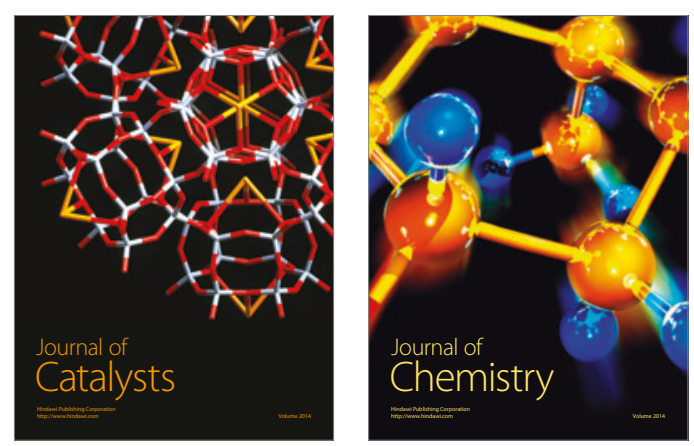
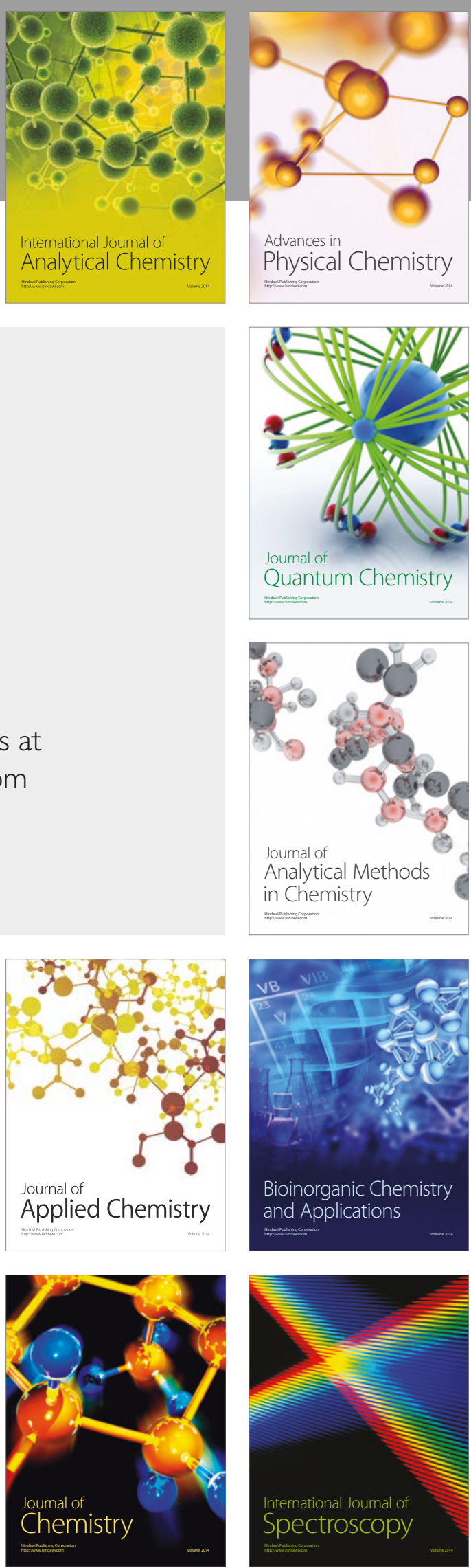